\title{
Bacterial chemotaxis towards aromatic hydrocarbons in Pseudomonas
}

\author{
Jesús Lacal, ${ }^{1}$ Francisco Muñoz-Martínez, ${ }^{1}$ \\ José-Antonio Reyes-Darías, ${ }^{1}$ Estrella Duque, ${ }^{1}$ \\ Miguel Matilla, ${ }^{2}$ Ana Segura, ${ }^{1}$ José-J. Ortega Calvo, ${ }^{3}$ \\ Celía Jímenez-Sánchez, ${ }^{3}$ Tino Krell ${ }^{1}$ and \\ Juan L. Ramos ${ }^{1 *}$ \\ ${ }^{1}$ Consejo Superior de Investigaciones Cientificas, \\ CSIC-EEZ, Department of Environmental Protection, \\ 18008 Granada, Spain. \\ ${ }^{2}$ Bio-liberis R\&D, Poligono Juncaril, C/Capileira 7, \\ 18220 Granada, Spain. \\ ${ }^{3}$ Consejo Superior de Investigaciones Cientificas, \\ CSIC-IRNASE, Seville, Spain.
}

\section{Summary}

Bacterial chemotaxis is an adaptive behaviour, which requires sophisticated information-processing capabilities that cause motile bacteria to either move towards or flee from chemicals. Pseudomonas putida DOT-T1E exhibits the capability to move towards ditferent aromatic hydrocarbons present at a wide range of concentrations. The chemotactic response is mediated by the McpT chemoreceptor encoded by the pGRT1 megaplasmid. Two alleles of mcpT are borne on this plasmid and inactivation of either one led to loss of this chemotactic phenotype. Cloning of mcpT into a plasmid complemented not only the mcpT mutants but also its transfer to other Pseudomonas conferred chemotactic response to high concentrations of toluene and other chemicals. Therefore, the phenomenon of chemotaxis towards toxic compounds at high concentrations is gene-dose dependent. In vitro experiments show that McpT is methylated by CheR and McpT net methylation was diminished in the presence of hydrocarbons, what influences chemotactic movement towards these chemicals.

\section{Introduction}

The rapid growth of the world's population has resulted in an increase in the presence of pollutants in the biosphere and among these, toxic and mutagenic xenobiotics represent a real threat to life (Kanaly and Harayama, 2010). Aromatic hydrocarbons are abundant in the biosphere due to the combustion of plant material, oil spills and the burning of petroleum derivatives. Mono-, di- and low molecular weight polycyclic aromatic hydrocarbons are easily degraded by microbes, whereas high molecular weight polycyclic aromatic hydrocarbons (PAHs) are often recalcitrant. Bioremediation uses the astonishing catabolic versatility of soil and aquatic bacteria to fight against pollutants. In addition, some strains are highly resistant to organic pollutants and have an enormous catabolic potential for biodegradation.

Although a large number of biodegradation protocols has been developed (as witnessed by some 2300 patents), the in situ outcomes of biodegradation are not always satisfactory. One of the reasons for the low biodegradation efficiency is the heterogeneous distribution of the pollutants or the reduced bioavailability of the target compounds (Pandey and Jain, 2002). Available information demonstrates that chemotaxis increases biodegradation efficiency in cases where the pollutant is heterogeneously distributed either in water or in soils (Marx and Aitken, 2000; Bhushan et al., 2004). In bacteria sensory recognition of a chemical gradient triggers a molecular signal transduction cascade, resulting in a modulation of flagellar activity, and in the directional movement of the bacteria either towards or away from stimulatory molecules (Alexandre et af., 2004; Wadhams and Armitage, 2004; Hazelbauer et al., 2008; Österberg et al., 2010; Lacal et al., 2010a). Although chemotaxis pathways in prokaryotes are known to vary, the main components of the chemotaxis signal transduction pathway are conserved and are made up of the ternary complex between methyl-accepting chemotaxis (MCPs) proteins, the sensor CheA protein and the adaptor protein (CheW or CheV). Signal recognition at the chemoreceptor level modulates CheA autophosphorylation activity and in turn transphosphorylation of the CheY response regulator, which acts on the flagellar motor.

While commensal microorganisms have only a few chemoreceptor, i.e. in $E$ coli there are four chemoreceptors (Hazelbauer et al., 2008), soil and aquatic bacteria possess a high number of MCPs; for instance, Pseudomonas sp. and Clostridum sp. possess more than 
20 chemoreceptors (Timmis, 2002; Hazelbauer et al., 2008). This enormous diversity of chemoreceptors is reflected in that many soil and aquatic bacteria are chemotactic to a wide range of different chemicals. Bacteria of the genus Pseudomonas have an enormous catabolic potential and a number of strains respond chemotactically to monocyclic and polycyclic aromatic hydrocarbons with nitro, amino or chloro substitutions (Grimm and Harwood, 1997; 1999; Parales et al., 2000; Gordillo et al., 2007; Iwaki et al., 2007; Liu and Parales, 2009), which makes this genus an ideal model system to study chemotaxis towards pollutants with the view to biotechnological exploitation.

We describe here a new form of chemotaxis through which bacteria are attracted directly to particles containing low or high concentrations of toxic compounds. The chemoreceptor involved in this phenotype in $P$. putida was identified as McPT and it is encoded on the selftransmissible pGRT1 (Rodríguez-Herva et al., 2007; Molina et al., 2011). There are two almost identical copies of $m c p T$ and inactivation of either of them leads to loss of the chemotactic phenotype indicating a clear gene-dose effect. The findings reported here and the molecular mechanisms underlying the chemotactic response to high concentrations of pollutants can have important implications in the design of new strategies to combat pollutants in heterogenously polluted sites.

\section{Results}

Pseudomonas putida strains exhibit different chemotactic phenotypes towards toluene

A set of Pseudomonas putida strains (Table 1) was analysed for their chemotactic response to toluene using agarose plug chemotaxis assays. In these qualitative assays an agarose drop containing the attractant is brought into contact with a bacterial suspension and toluene diffuses into the bacterial culture creating a concentration gradient. The accumulation of bacteria around the agarose plug is a measure of chemotaxis. Two patterns of accumulation were observed: one in which cells accumulated at a distance of 1-2 mm from the toluene plug, which was termed moderate taxis ( $P$. putida F1 and KT2440, Fig. 1). The second pattern was characterized by an accumulation of cells on the toluene-containing plug. This phenotype was exclusively observed for $P$. putida DOT-T1E. This phenotype was termed strong chemotaxis or hyperchemotaxis. This behaviour was observed for toluene concentrations between $0.01 \%(\mathrm{v} / \mathrm{v})$ and $50 \%(\mathrm{v} / \mathrm{v})$.

Chemotaxis was also studied using capillary assays. In this assay a capillary was filled with agarose containing $1 \%(\mathrm{v} / \mathrm{v})$ toluene, and the open end of the capillary was submerged into a bacterial solution. We found that P. putida DOT-T1E cells accumulated at the entrance to the capillary, whereas $P$. putida KT2440 formed a cloud at

Table 1. Strains and plasmids used in this study.

\begin{tabular}{|c|c|c|}
\hline Strains & Features & References \\
\hline P. putida DOT-T1E & Tolf $^{\text {, }}$ wild-type, Rifi & Ramos et al. (1995) \\
\hline P. putida DOT-T1E-100 & $\mathrm{TOl}^{\mathrm{S}}$ derivative of DOT-T1E, Riff & Rodríguez-Herva et al. (2007) \\
\hline P. putida DOT-T1E $\triangle \mathrm{C}$ & $\mathrm{Tol}^{-}$derivative & This laboratory \\
\hline P putida DOT-T1E-PS28 & Tol $^{8}$, Rif $^{\mathrm{B}}, \mathrm{pGRT} 1, \operatorname{tg} V: \because a p h A-3, \mathrm{Km}^{\mathrm{B}}$ & Rojas et al. (2003) \\
\hline P. putida DOT-T1E $\Delta m c p T 1$ & Tol $^{\mathrm{s}}, \mathrm{Rif}^{\mathrm{R}}, \mathrm{Km}^{\mathrm{R}}$, pGRT1, mcpT-1::pChesi & This work \\
\hline P. putida DOT-T1E $\triangle m c p T 2$ & Tol $^{\mathrm{s}}, \mathrm{Rif}^{\mathrm{P}}, \mathrm{Km}^{\mathrm{R}}, \mathrm{pGRT1}$, mcpT-2::pChesi & This work \\
\hline P. putida DOT-T1E $\triangle m c p T 1, m c p T 2$ & $\mathrm{Rif}^{\mathrm{F}}, \mathrm{Km}^{\mathrm{R}}, \mathrm{Gm}^{\mathrm{R}}$, mcpT1:pChesiKm mcp T2: pChesiGm & This work \\
\hline P. putida KT2440 crc- (ttgV::aphA-3) & pGRT1:: $\triangle \operatorname{tg} V$ & Rodríguez-Herva et al. (2007) \\
\hline P. putida $\mathrm{F} 1$ & $\mathrm{Tol}^{\mathrm{s}}$ & This laboratory \\
\hline P. putida KT2440 & mt-2 pWWO cured, Tols - & Timmis (2002) \\
\hline P. putida MT53 (pWW53) & $\mathrm{Tol}^{\mathrm{s}} \mathrm{ph}$ & Yano et al. (2007) \\
\hline P. putida pCAR1 & $\mathrm{Tol}^{\mathrm{S}}$ & Miyakoshi et al. (2007) \\
\hline \multicolumn{3}{|l|}{ Plasmids } \\
\hline pGRT1 & Large self-transmissible plasmid & Rodríguez-Herva et al. (2007) \\
\hline 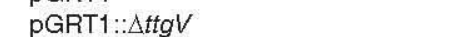 & pGRT1, $\operatorname{tg} V:: a p h A-3, \mathrm{Km}^{\mathrm{R}}$ & Rojas et al. (2003) \\
\hline PGRT1::Аm cpT & pGRT1, mcpT1::pCHESI, Km ${ }^{R}$ & This work \\
\hline pGRT1:: $\triangle \mathrm{m}$ cpT2 & mсрT2: $\mathrm{pCHESI}, \mathrm{Km}^{\mathrm{R}}$ & This work \\
\hline PBBR1MCS-5 & oriT RK2, Gm & Kovach et al. (1995) \\
\hline pJL-McpT1 & mcpT, gene cloned in pBBR1MCS-5, $\mathrm{Gm}^{\mathrm{R}}$ & This work \\
\hline pJL-McpT S183P & mcpT, S183P cloned in pBBR1MCS-5, Gm ${ }^{R}$ & This work \\
\hline pCHESISKm & $\mathrm{mob}^{+}$, ColE1 replicon, $\mathrm{Km}^{\mathrm{P}}$ & Llamas et al. (2003) \\
\hline pJL-P $P_{\text {mOPT }}$ & $\mathrm{Tc}^{\mathrm{R}}$, lacZ:: $\mathrm{P}_{\text {map }}$ promoter, IncW & This work \\
\hline pET200/DTOPO & $\mathrm{Km}^{\mathrm{F}}$, protein expression vector & Invitrogen \\
\hline pJL-McpT-FullLength & $\mathrm{pET} / \mathrm{TOPO}$ containing the $m c p T$ gene, $\mathrm{Km}^{\mathrm{R}}$ & This work \\
\hline
\end{tabular}

$\mathrm{Gm}^{R}, \mathrm{Km}^{R}$, Rif ${ }^{R}$ and $\mathrm{Sm}^{R}$ indicate resistance to gentamicin, kanamycin, rifampicin and streptomycin respectively. In addition, Tol ${ }^{+}$indicates that the strain grows on toluene, whereas Tol indicates that the strain cannot use toluene as the sole carbon and energy source. Tol $^{\mathrm{P}}$ and $\mathrm{Tol}^{\mathrm{s}}$ stand for tolerance and sensitivity, respectively, to $>0.3 \%(v / v)$ toluene in liquid medium. 
A

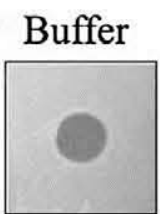

Succinate

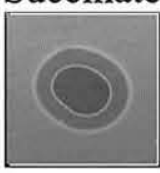

F1
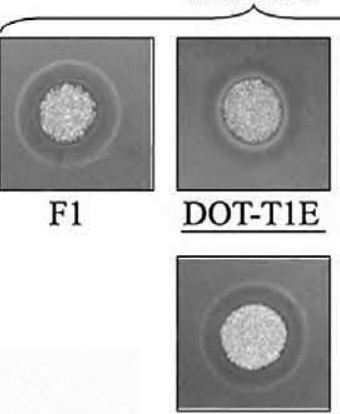

DOT-T1E

100

B

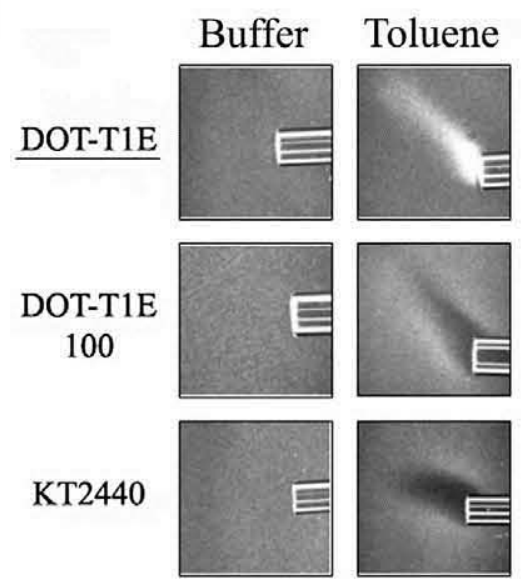

Fig. 1. Chemotactic behaviour of different strains of $P$. putida towards toluene.

A. Agarose plug assays of strains F1, DOT-T1E and KT2440. Strain DOT-T1E-100 is a derivative of DOT-T1E lacking the large, self-transmissible plasmid pGRT1 (Rodríguez-Herva et al., 2007). Strain KT2440 (pGRT1:: $\Delta t t g V$ ) has the ttgV gene disrupted by a kanamycin cassette. Strains presenting the hyperchemotaxis phenotype are underlined. Controls show the response of strain DOT-T1E to buffer and succinate.

B. Capillary assays of strains DOT-T1E, DOT-T1E-100 and KT2440 towards toluene which was immobilized in the capillary at a concentration of $10 \%$ ( $\mathrm{V} / \mathrm{V}$ ).
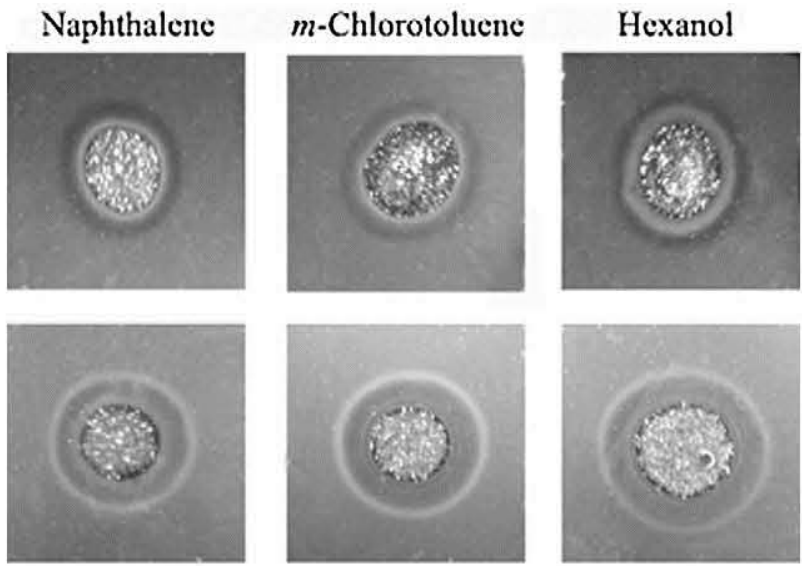

Benzonitrile
p-Chlorotoluene

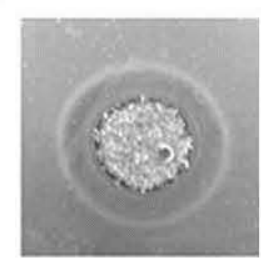

Ethylbenzene

Fig. 2. Chemotactic response of $P$. putida DOT-T1E to different hydrocarbons. Agarose plug assays using a chemoattractant concentration of $1 \%(\mathrm{v} / \mathrm{v})$. Compounds shown in the upper row cause the hyperchemotaxis phenotype, whereas compounds in the lower row provoke a moderate chemotaxis phenotype. The full range of compounds analysed is shown in Table 2 .

a defined distance (Fig. 1). The chemotactic response to high concentrations of aromatic compounds of $P$. putida DOT-T1E was observed when cells were grown in rich Luria-Bertani (LB) medium or in M9 minimal medium with different carbon sources in the absence and in the presence of toluene, what indicates that this chemotactic response is of constitutive nature. This chemotactic response was also observed with Pseudomonas putida DOT-T1E $\Delta C$ (Table 1) a mutant strain that harbours a knock out in the todC gene and, therefore, cannot metabolize toluene. This suggests that the strong chemotactic response towards toluene is independent of the metabolism of the chemoattractant. We used 36 different hydrocarbons, at a concentration of $1 \%(\mathrm{v} / \mathrm{v})$, to assess the spectrum of attractants that give rise to the chemotaxis phenotype. P. putida DOT-T1E exhibited strong chemotaxis in response to $70 \%$ of the tested compounds (Table 2, Fig. 2). For $10 \%$ of the compounds no or weak

Table 2. Different phenotypes observed for the chemotaxis of P. putida DOT-T1E towards different hydrocarbons.

None or very weak chemotaxis

benzene, styrene, $p$-xylene, $p$-nitrotoluene

Moderate chemotaxis

Benzene derivatives:

chlorobenzene, nitrobenzene, ethylbenzene

fluorobenzene, benzonitrile

Toluene derivatives:

p-ethyltoluene, $p$-chlorotoluene

p-bromotoluene

\section{Hyperchem otaxis}

Benzene derivatives: propylbenzene, butylbenzene

Toluene and singly substituted derivatives:

toluene, o-xylene, $m$-xylene, o-iodotoluene

$m$-iodotoluene, $p$-iodotoluene, o-toluidine

$m$-toluidine, $p$-toluidine, $o$-chlorotoluene

$m$-chlorotoluene, $m$-bromotoluene

$m$-fluorotoluene, $p$-fluorotoluene, o-fluorotoluene

$m$-nitrotoluene, o-nitrotoluene

Multiply substituted benzene derivatives

1,2,4-trimethylbenzene, 1,3,5-trimethylbenzene

2,3-dimethylphenol

Biaromatics:

naphthalene, 1,2,3,4-tetrahidronaphthalene 
taxis was observed, whereas the remaining $20 \%$ showed moderate taxis. P. putida DOT-T1E also showed the strong chemotactic response when the agarose plug was replaced with undiluted crude oil pellets collected from the Spanish sea shores after the sinking of the Prestige oil tanker (data not shown).

\section{The pGRT1 plasmid confers strong chemotaxis to Pseudomonas putida towards toluene}

We showed before that the solvent-tolerant character of the DOT-T1E strain was due to the presence of a series of efflux pumps that expel solvents to the outer medium (Rojas et al., 2003). From a quantitative point of view the most relevant pump is TtgGHI, encoded on the pGRT1 self-transmissible plasmid present in this strain (Rojas et al., 2003; Rodríguez-Herva et al., 2007). To test whether the extrusion of the chemoattractant was the reason for the strong chemotatic phenotype, a mutant strain, DOT-T1E-PS28 (Rojas et al, 2003), with a nonfunctional TtgGHI pump, was used. Agarose plug assays revealed that the strong chemotactic behaviour of mutant DOT-T1E-PS28 was similar to that of its parental strain, suggesting that the TtgGHI efflux pump is not the primary cause of the strong chemotaxis phenotype.

To test whether the molecular determinant(s) for strong chemotaxis was/were associated with the pGRT1 plasmid, agarose plug and qualitative capillary chemotaxis assays of a plasmid-less variant of DOT-T1E, known as DOT-T1E-100, were carried out. These assays revealed that the plasmid-less strain exhibited the moderate chemotaxis phenotype and that it behaved similarly to the KT2440 and F1 strains (Fig. 1).

Rodríguez-Herva and colleagues (2007) showed that pGRT1 can be transferred to other Pseudomonas strains, for example KT2440. To verify whether pGRT1 is responsible for the strong chemotaxis phenotype, $P$. putida KT2440 (pGRT1: $\Delta \operatorname{tg} V$ ) was analysed with regard to strong chemotaxis. Interestingly, this strain exhibited the same strong chemotaxis phenotype as DOT-T1E (Fig. 1), providing evidence for the role of pGRT1 borne genes in strong chemotaxis.

The sequence analysis of the low-copy-number (1-3 copies per cell) $133 \mathrm{kbp}$ pGRT1 plasmid (Fig. S1) revealed the presence of two ORFs, which encode for methyl-accepting chemotaxis proteins (MCP), that we termed McpT1 and McpT2, and which vary in only one amino acid at position 201 (glutamic acid or alanine). To provide unequivocal evidence of the potential role of these genes in the chemotactic behaviour, mutants were generated by site-directed mutagenesis (see Experimental procedures). The inactivation of either of the alleles resulted in the loss of the strong chemotactic behaviour resulting in strains that only exhibited the moderate

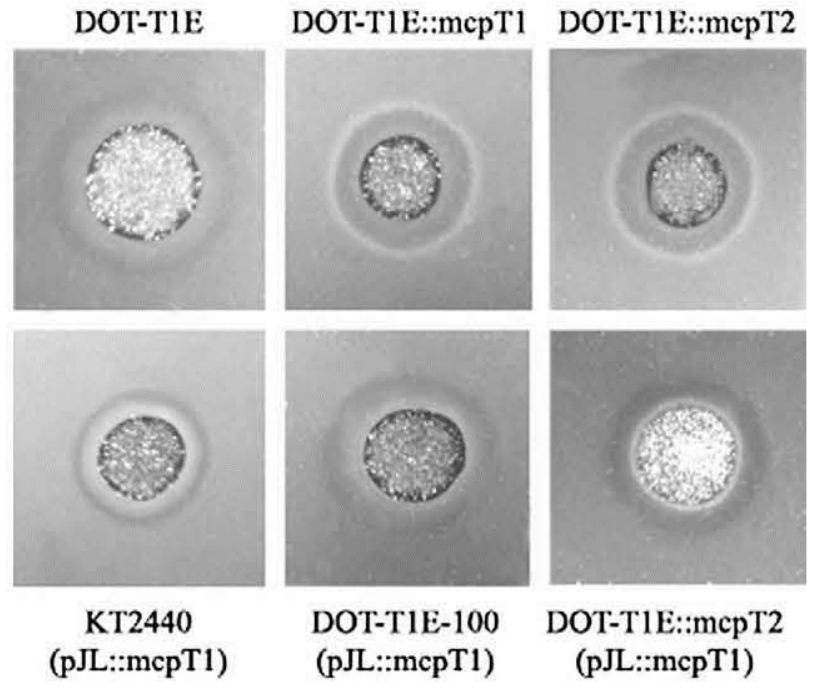

Fig. 3. The McpT chemoreceptor is the molecular determinant for hyperchemotaxis. Agarose plug assays of $P$. putida DOT-T1E, DOT-T1E::mcpT1, DOT-T1E::mcpT2; P. putida KT2440, DOT-T1E100 complemented with pJL::MCPT1.

chemotaxis phenotype (Fig. 3). To quantify the response of the mcpT mutants to toluene and succinate - used as control - quantitative capillary assays were performed. To this end a modified version of the assays described by Adler (1973) was employed (see Experimental procedures). Buffer containing different concentrations of the chemoattractant is placed into the capillary, which is then brought into contact with a bacterial suspension. After and exposure of $10 \mathrm{~min}$, the contents of the capillary are collected and the number of colony forming units is quantified.

In an initial series of experiments the response of strains DOT-T1E, its plasmid free derivative DOT-T1E100 and a DOT-T1E mutant deficient in the genes mcpT1 and mcpT2 were analysed. As shown in Fig. 4A, in the absence of toluene (buffer present in the capillary) these three strains were characterized by a similar motility. The DOT-T1E wild-type strain showed an initial response at $1 \%$ of toluene and a significant increase in the magnitude of response at $5 \%$ and $10 \%$ toluene was observed. For the mutant strains deficient in mcp T1 and mcp T2 an initial slight response was also observed at $1 \%$ toluene but the increase in taxis at $5 \%$ and $10 \%$ toluene was significantly modest as compared with the wild-type strain. Surprisingly, the DOT-T1E-100 strain, lacking in the entire plasmid, did not respond at $1 \%$ toluene and less cells accumulated in the capillary containing $5 \%$ and $10 \%$ toluene than in the buffer control. The difference in responses observed in the mcpT double mutant and the plasmid-less strain is likely due to the presence or, respectively, absence of the plasmid encoded TtgGHI efflux pump, which has been shown to play a key role in 

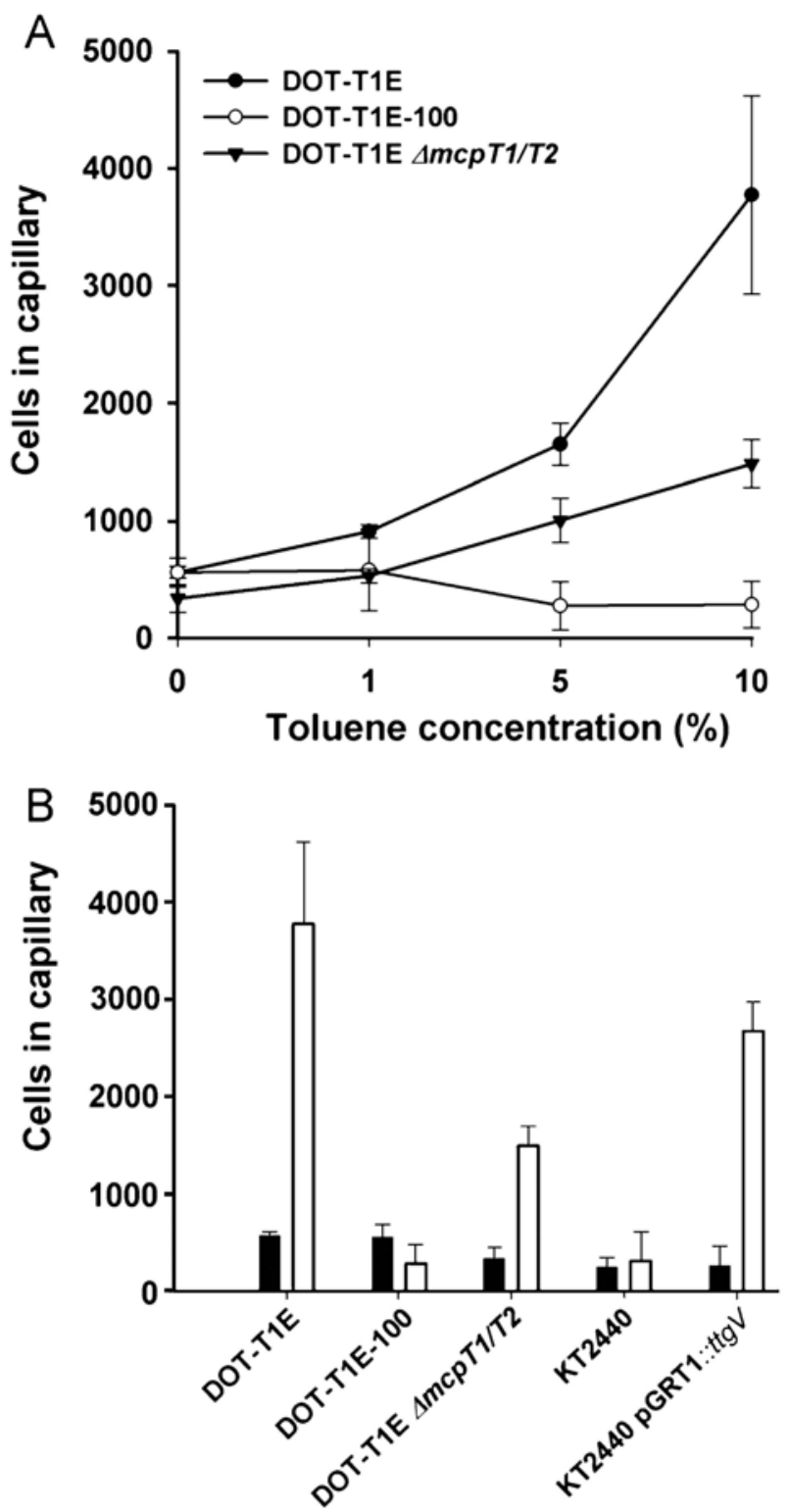

Fig. 4. Quantification of the chemotactic response towards toluene of different Pseudomonas putida strains. Shown are data obtained from quantitative capillary assays.

A. The chemotactic responses of $P$. putida DOT-T1E $(\bullet), P$. putida DOT-T1E-100 $(O)$ and of a double mutant ( $P$. putida DOT-T1E $\Delta m c p T 1 / T 2)(6)$ to various concentrations of toluene (\%) in a capillary assay. Results represent the means from at least three independent experiments conducted in triplicates; the error bars indicate standard errors.

B. Quantitative chemotactic response of different strains of Pseudomonas putida to toluene (10\% v/v). Filled bars represent accumulation of cells in capillaries containing minimal medium without attractant and open bars represents accumulation of cells in capillary tubes containing $10 \%(\mathrm{v} / \mathrm{v})$ toluene. Results represent the means from at least three independent experiments conducted in triplicates; except measurements of strain KT2440 which represents a single experiment conducted in triplicates. conferring resistance to elevated concentrations of toluene. Although the agarose plug assays reported above demonstrated that TtgGHI is not the primary determinant of the strong chemotaxis phenotype, these quantitative measurements suggest that the resistance conferred by this efflux pump increases the magnitude of the response.

To provide further grounds on this hypothesis we carried out further assays and reasoned that the cloning of an mcpT allele in a medium-copy-number plasmid should be sufficient enough to provide the strong chemotactic behaviour to a recipient host. To test this hypothesis the mcpT1 allele was cloned into the broad-host range medium-copy-number (15-30 copies per cell) vector pBBRMCS-5 and the resulting plasmid, pJL:: $m c p T$ was introduced into different $P$. putida backgrounds, namely, the plasmid-free derivative of DOT-T1E, DOT-T1E-100, the mcpT1 and mcpT2 mutants and P. putida KT2440. In all cases the acquisition of the pJL::mcpT plasmid conferred the strong chemotactic response to the host (Fig. 3). A second series of quantitative chemotaxis assays was aimed at quantifying the response of strain KT2440 and its derivative harbouring plasmid pGRT1::ttg $V$ to $10 \%$ toluene. For these experiments a derivative of plasmid pGRT1 was used, which contained a kanamycin resistance cassette inserted into the $t t g V$ gene. Strain KT2440 showed a motility comparable to that of the plasmid-less version of DOT-T1E (Fig. 4B). It should be noted that both strains lack the TtgGHI efflux pump. However, taxis of strain KT2440 pGRT::ttgV was largely increased as compared with its parental strain, which illustrates that the transfer of plasmid pGRT1 to other Pseudomonas strains causes an increase in the chemotactic attraction towards toluene.

A BLAST search using the McPT as query revealed that McpT homologues (>99\% sequence identity) are exclusively present as monocopy on catabolic plasmids, i.e. pCAR1 of Pseudomonas resinovorans (Miyakoshi et al., 2007), the TOL plasmid pWW53 of $P$. putida (Yano et al., 2007) and the plasmid pMAQU02 of Marinobacter aquaeolei VT8. Interestingly, these three homologues are identical amongst them and share $99.8 \%$ sequence identity ( 1 amino acid change) with McpT of pGRT1.

Plasmid pCAR1 was transferred into $P$. putida KT2440 (Miyakoshi et al., 2007), and the resulting strain, P. putida KT2440 (pCAR1), assayed with regards to its chemotactic response to toluene. We found that KT2440 (pCAR1) exhibited only the moderate chemotaxis phenotype. The $108 \mathrm{kbp}$ plasmid pWW53 is a well-studied TOL plasmid (Yano et al., 2007) present in P. putida MT53. Pseudomonas putida MT53 containing pWW53 exhibited only the moderate chemotactic response towards toluene (not shown). This set of results support that the McpT protein 
plays a key role in the chemotactic response to toluene and that its effect is gene dose-dependent.

\section{Expression of the mcpT1 promoter is constitutive}

The mcp $T$ genes in pGRT1 are monocistronic units, and the expression of the genes was studied using a fusion of the $\mathrm{P}_{\text {mcot }}$ promoter region to 'lacZ in pMP220. $\beta$-Galactosidase assays were carried out with DOT-T1E and DOT-T100 cells grown on M9 minimal medium with glucose, in the absence and in the presence of toluene. In both genetic backgrounds, the level of activity in the absence and the presence of aromatic hydrocarbons was found to be $100 \pm 10$ Miller units regardless of the growth phase, indicating that the $m c p T$ genes are constitutively expressed.

\section{Methylation of the chemoreceptor McpT by} Pseudomonas putida CheR

The above data support that the mcpT gene mediates the strong chemotactic response. We have assessed whether McpT has the typical characteristics of a chemoreceptor. One of such characteristics is the capability to be methylated by a CheR methyltransferase and the capability of receptor ligands to modulate this activity. To this end, the mcp T was cloned in pET200/D-TOPO, expressed in E. coli (Fig. S1) and the corresponding membranes were purified. Control membranes devoid of McpT were also prepared using cells that only bear pET200/D-TOPO. Subsequently, methylation assays were performed using purified CheR (PP3760) from $P$. putida DOT-T1E in the absence and in the presence of toluene.

With McpT-free membranes, regardless of the presence of toluene and regardless of addition of CheR, low levels of methylation were found (Fig. 5). With the McpT enriched membranes, the methylation signal was high when CheR was added. Interestingly, in the same assay but in the presence of toluene, the level of methylation was $20-30 \%$ lower than in the absence of toluene. To confirm that McpT is indeed methylated by $\mathrm{CheR}$, aliquots of radiolabelled methylated McpT-containing membranes were subjected to SDS-PAGE, the gels were stained with Coomassie, fixed and autoradiographied. The results unequivocally confirmed that McpT was the protein methylated by CheR (Fig. 5A).

\section{A helix-break point mutation in the McpT ligand binding domain inactivates Mcp T}

To further provide insights into the role of McpT in the chemotactic response a site directed mutant was constructed based on McpT sequence analysis. The analysis of the 552 amino acid sequence of McpT using DAS

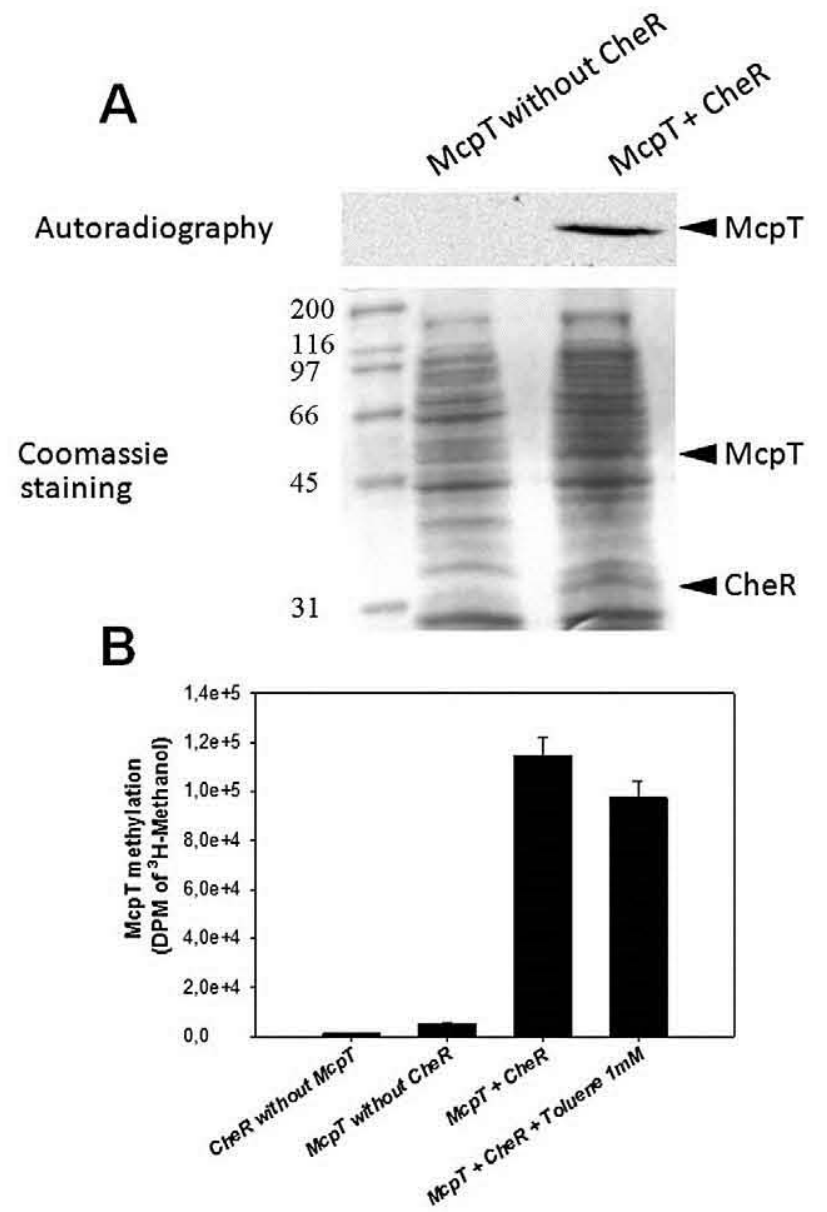

Fig. 5. Methylation of McpT by CheR.

A. E. coli membranes containing recombinantly expressed McpT were submitted to methylation assays in the presence and absence of purified recombinant CheR of $P$. putida KT2440. For theses assays tritium labelled $\mathrm{S}$-adenosylmethionine was used (further details are found in Experimental procedures). Shown is a Coomassie stained SDS-gel of the reaction $\mathrm{mix}$ and the bands corresponding to McpT and CheR are indicated by an arrowhead. The autoradiograph shows the molecular weight region corresponding to McpT. In the absence of CheR no methylation was observed.

B. Methylation of McpT followed by the vapour-phase equilibrium procedure (see Experimental procedures). The left column corresponds to assays with McpT-free control membranes - but with P. putida CheR. The second column from the left corresponds to an assay with McpT enriched membranes but without CheR respectively. The following two assays correspond to methylation of McpT by CheR in the absence and presence of $1 \mathrm{mM}$ toluene. Samples were taken after $20 \mathrm{~min}$ incubation.

(Cserzö et al., 1997) revealed the presence of two transmembrane regions flanking the putative periplasmic ligand binding domain (LBD) that spans from residue G27 to M202 (Fig. S2). The prediction of the secondary structure of this domain using a consensus method indicated the presence of four $\alpha$-helices (Deleage et al., 1997), which is consistent with a 4-helix bundle arrangement typical of TarH-type domains (Yeh et al., 1996). In TarH 
the final $\alpha$-helix of the LBD extends into the membrane forming the second transmembrane region. Ligand binding induces a piston-type shift of this transmembrane helix, which is thought to be the regulatory stimulus altering CheA autophosphorylation (Ottemann et al., 1999).

On the basis of the in silico data, we reasoned that a mutant of McpT in which Ser183 (an amino acid located in the middle of the final helix of the LBD) is replaced by the helix-breaking amino acid; proline could interfere with receptor functioning. The mutant variant was generated by site-directed mutagenesis, cloned in pBBMRCS5 to yield pJL::mcpTS183P and transferred to KT2440, DOT-

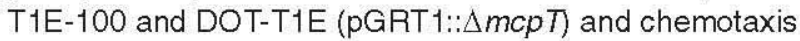
assays were done. In contrast with the strain bearing the native McpT protein, the set of constructs with the McpTS183P mutant showed only the moderate chemotaxis phenotype. Therefore, a single point mutation, which, most likely, interrupts transmembrane signalling resulted in the inactivation of McpT and has a major phenotypic effect in the chemotactic response to toluene.

\section{Discussion}

It is generally assumed that throughout evolution microorganisms developed effective mechanisms that assist them to regulate cellular functions in response to environmental changes (Taguchi et al., 1997). Of these, chemotaxis is one of the best studied spatial-temporal behaviours required for the colonization of niches. One of the remarkable features of chemotaxis is that bacteria sense a wide range of chemicals at different concentrations and respond to them. In this regard, $P$. putida bearing the NAH7 plasmid responds to naphthalene using the NahY protein as a chemoreceptor (Grimm and Harwood, 1999) and $P$. fluorescens strain KU-7 responds to 2-nitrobenzoate through a plasmid-encoded $\mathrm{NbaY}$ chemoreceptor (Iwaki et al., 2007). A limited number of studies have shown that chemotactic movement enhances the mineralization rate of heterogeneously distributed toxic organic compounds (Marx and Aitken, 2000; Bhushan et al, 2004). In this study we have shown that a number of $P$. putida strains respond chemotactically to different aromatic hydrocarbons, and that two types of behaviour were evident in agarose plug assays; one was the formation of rings at a defined distance from the chemical, a phenomenon we term moderate chemotaxis; whereas the other is an unusual new phenotype, termed strong chemotaxis in which $P$. putida DOT-T1E cells are attracted to the agarose plug in which the pollutant is embeded. The strong chemotactic response was also observed with crude oil, reinforcing the potential use of bacteria displaying the strong chemotactic behaviour for the practical treatment of polluted sites.
Pseudomonas putida DOT-1E is considered an extremophile in the sense that it grows in the presence of concentrations of solvents that impede the growth of most microbes. The strong chemotactic response adds a new remarkable property to DOT-T1E, i.e. the ability to swim towards both low and very high concentrations of pollutants. Interestingly, the key genes responsible for these two phenotypes (solvent tolerance and hyperchemotaxis) are encoded on the 133-kb self transmissible pGRT1 plasmid (Rodríguez-Herva et al., 2007). Another relevant feature is that both solvent tolerance and chemotactic responses are gene-dose dependent. In this study we showed that two copies of the chemoreceptor mcpT gene (the two alleles differ in a single nucleotide) are required for the strong chemotactic response. These copies appear in a $4.5 \mathrm{~kb}$ duplicated fragment of pGRT1 (Fig. S1), and inactivation of either of these genes results in the loss of the strong chemotactic phenotype. Furthermore, cloning of either of the mcp $T$ alleles in a mediumcopy number vector is sufficient to confer the strong chemotactic phenotype to different strains of $P$. putida. It is also of interest to note that a plasmid-free derivative of DOT-T1E, strain DOT-T1E-100, still retained the moderate chemotactic response to toluene, which indicated that toluene and related aromatics can be also be detected by one or more chromosomally encoded chemoreceptors. A type of hyperchemotaxis response was previously described in Halobacterium salinarum and $B$. subtilis regarding their response to the oxygen levels in the medium (Aono et al., 2002; Kristich and Ordal, 2004). Two types of chemoreceptors, HemA and HtrVIII in H. salinarum, and HemA-Bs and OI3545 in B. subtilis were found to be required for this hyperchemotactic response (Brooun et al, 1998; Zhulin, 2001; Delalez and Armitage, 2009). Since McpT1 and McpT2 in P. putida differ in only one amino acid, we suggest that bacterial hyperchemotaxis is the result of the participation of more than one chemoreceptor, or mediated through a gene-dose dependent effect.

In bacterial chemotaxis, the chemoreceptor proteins or MCPs are reversibly methylated as a way of receptor adaptation to varying concentrations of chemoattractants. In Escherichia coli and Salmonella typhimurium, the enzyme responsible for this modification is CheR (Boukhvalova et al., 2002), which catalyses the transfer of methyl groups from S-adenosylmethionine (SAM) to specific glutamyl residues in the cytosolic domain of MCPs. Binding of chemoattractants at the periplasmic domain triggers a conformational changes, which modulates CheA activity (Chervitz and Falke, 1996) and which in parallel alters receptor methylation by CheR (Brooun et al., 1998). Receptor methylation is thought to generate a molecular stimulus that travels back to the periplasmic domain and resets the receptor complex to its null state by 
reversal of the conformational change generated by ligand binding (Lai et al., 2006), which is a mechanism leading to receptor adaptation. It is known that chemoattractants in $E$. coli cause an increase in net methylation whereas in Bacillus subtilis attractants cause net reduction of methylation (Alexander and Zhulin, 2007). Here we show that McpT in P. putida is specifically methylated by CheR and that amount of methylation of McpT was moderately but reproducibly reduced in the presence of toluene. This supports that toluene interacts with McpT and indicates that the methylation mechanism in P. putida might resemble that described for $B$. subtilis. Inhibition of McpT methylation could affect the autophosphorylation of CheA that in turn modulates CheY phosphorylation level and subsequently the functioning of the flagella motor.

To place these observations in a large context we should note that when bacteria are exposed to compounds which are toxic but which at the same time can be used as a carbon source, microorganisms face the obvious dilemma as to flee from these compounds or to fight and use them. The decision-marking process is based on the action of different signal transduction proteins. Some bacteria have evolved to flee whereas others have evolved to resist and to use the toxic compounds. Among the last group of strains $P$. putida DOT-T1E is a well characterized example and the data reported here largely contribute to the mechanisms which form the basis for this 'decision'. Strain DOT-T1E can use toluene as its sole carbon source, which is due to the action of the TOD pathway which is under the control of the TodS/TodT two-component system. The sensor kinase TodS was found to bind toluene and upregulate gene expression accordingly (Lacal et al., 2006; Busch et al., 2007; 2009). In addition this strain possesses the $\mathrm{TtgGH}$ efflux pump which expulses toluene (Rojas et al., 2003). The action of this pump was found to be the ultimate determinant for solvent tolerance in this microorganism. The TtgV repressor binds toluene and upregulates pump expression accordingly (Guazzaroni et al., 2005). Both toluene sensor proteins are present in the cytosol, but the affinity of TodS for toluene is around 2 orders of magnitude higher than that of TtgV. This is consistent with the notion that at low toluene concentration the bacteria degrades toluene and only at high toxic concentration the TtgV mediated upregulation of the $\mathrm{tg}$ GHI operon.

In the present work we have characterized the primary player which can be associated to the flee-part of the dilemma. Aromatic hydrocarbons are for some bacteria chemoattractants, whereas for others chemorepellents. McpT was shown to mediate a strong chemoattractant response. Interestingly, two of the three toluene binding signal transduction proteins (McpT and TtgVTtgGHI) are present on the plasmid pGRT1. This might indicate that the hyperchemotaxis reported here requires mechanisms which guarantee the survival of the bacteria in the presence of the chemoattractant.

Regardless of the molecular mechanism of McpT functioning, it is interesting to note that McpT responds to a wide range of chemicals, that the mcpT genes are plasmid borne and they can be horizontally transferred to other microbes. Genome analysis reveals that almost identical McpT homologues are present in a series of other bacterial plasmids, which are present in strains that are able to degrade aromatic hydrocarbons. This is consistent with the notion that the strong chemotaxis phenotype is not restricted to strain DOT-T1E but might have co-evolved in several bacteria with the capacity to degrade aromatic hydrocarbons. From a biotechnological perspective in bioremediation strong chemotaxis can be exploited to allow bacteria to move towards pollutants and achieve a more efficient bioremediation outcome in heterogeneously polluted sites.

\section{Experimental procedures}

\section{Growth of bacterial strains}

Pseudomonas putida wild-type and mutant strains were grown in $\mathrm{M} 9$ minimal medium supplemented with $10 \mathrm{mM}$ succinate, in the absence (non-induced conditions) or in the presence of $1.5 \mathrm{mM}$ toluene (induced). Appropriate antibiotics were added when required. Bacteria and plasmids used are listed in Table 1. Antibiotics were added to the culture medium to reach $50 \mu \mathrm{g} \mathrm{ml}^{-1} \mathrm{Gm}$ or $\mathrm{Km}$ and $10 \mu \mathrm{g} \mathrm{ml}^{-1}$ in the case of Rif, Tc and Sm.

\section{Chemotaxis assays}

Agarose plug assays were carried out as previously described (Parales et al, 2000). In short, the mixture for generation of the agarose plug contained $2 \%$ (W/N) lowmelting-temperature OmniPur agarose (EMD), chemotaxis buffer $\left(40 \mathrm{mM} \mathrm{K}_{2} \mathrm{HPO}_{4} / \mathrm{KH}_{2} \mathrm{PO}_{4}, 0.05 \%\right.$ (w/v) glycerol, $10 \mathrm{mM}$ EDTA, pH 7.0), the chemoattractant at several concentrations ranging from $0.01 \%$ to $50 \%(\mathrm{~V} / \mathrm{V})$ and trace amounts of Coomassie blue to provide contrast. A $5 \mu \mathrm{l}$ drop of this mixture was placed on a microscope slide on top of a coverslip supported by two plastic strips and placed to form a chamber. Cells grown as described above were harvested when cell density was $0.3-0.7$ at $660 \mathrm{~nm}$, resuspended in chemotaxis buffer to reach an $\mathrm{OD}_{660}$ of 0.7 , and then introduced into the chemotaxis chamber to surround the agarose plug. As control for this series of assays, plugs without chemicals were used as negative control, whereas plugs with $6 \mathrm{mM}$ succinate were used as a positive control, because $P$. putida responds chemotactically to this dicarboxylic acid (Lacal et al., 2010b).

For qualitative capillary assays, each capillary was filled with $1 \%(w / N)$ low-melting-temperature agarose in chemotaxis buffer and $2.5-10 \%(\mathrm{~V} / \mathrm{V})$ of the attractant. Freshly grown cells were resuspended in chemotaxis buffer to an $\mathrm{OD}_{660}$ of $0.1 \pm 0.05$ and placed into an observation chamber formed by a microscope slide and a coverslip. The capillary contain- 
ing the attractant was then inserted into the cell suspension. Cells were observed under $400 \times$ magnification in a Zeiss microscope coupled to a photographic camera or a video camera.

\section{Quantitative capillary assay}

A modified version of the capillary test described earlier (Adler, 1973) was used to quantify chemotaxis. In short, cells were grown in minimal medium (Iwaki et al., 2007) to a turbidity, at $600 \mathrm{~nm}\left(\mathrm{OD}_{600}\right)$ of $0.3-0.8$. Subsequently, cells were diluted in the same medium to an $\mathrm{OD}_{600}$ of approximately 0.08 . About $0.1 \mathrm{ml}$ of this suspension was placed into a small chamber formed by placing two capillary tubes (volume $1 \mu \mathrm{l}$ ) (Microcaps, Drummond, Broomalll, PA, USA) in parallel on a microscope slide. Another capillary tube, heat-sealed at one end, containing the chemoeffector solution, was immersed in the cell suspension at its open end. The system was then closed with a glass coverslip avoiding any formation of air bubbles in the chamber. The chemoeffector solution in the capillary contained minimal medium supplemented with different concentrations of toluene [1, 5 and $10 \%(\mathrm{~V} / \mathrm{N})]$. After $10 \mathrm{~min}$ incubation, the number of cells in the capillary was quantified by colony-forming units on minimal medium M9 supplemented with $10 \mathrm{mM}$ of succinate as a carbon source.

\section{Verification of the presence of pGRT1 by PCR}

The presence or absence of pGRT1 in different bacterial strains was verified by polymerase chain reaction (PCR) using the oligonucleotides 5'-CTGATCAGGCTGTAAAGT GGCTGG-3' and 5'-GCCCTGCGTTTTAGCGATGGCTTC-3', which are fragments of the plasmid-encoded genes IspA and orf156 respectively (Rodríguez-Herva et al., 2007). A large bacterial colony was resuspended in $10 \mu \mathrm{l}$ of water, heated at $100^{\circ} \mathrm{C}$ for $10 \mathrm{~min}$ and centrifuged at $20000 \mathrm{~g}$ for $2 \mathrm{~min}$. Three microlitres of the resulting supernatant was used as template for PCR amplifications using standard conditions. Reaction products were analysed on $0.8 \%$ (w/v) agarose gels. The amplified product from a pGRT1 containing strain is $2314 \mathrm{bp}$ in length.

\section{Construction of the PmcpT promoter fusion and determination of $\beta$-galactosidase activity}

The mcpT promoter was amplified using McpTP1 (5' ACCTGACGGAATTCAGGTCTTGTATT-3') and McpTP2 (5' TGTCACCTCATCTGCAGATATTTCAATGTG-3') primers containing EcoRI and Pstl sites respectively. The $153 \mathrm{bp}$ PCR product was digested with EcoRI and Pstl and cloned into likewise digested pMP220. As a result, the $\mathrm{P}_{\text {mcps }}$ promoter was fused to ' $/ a c Z$ in pMP220 to produce $\mathrm{pJL}:: \mathrm{P}_{\text {mop }}$. To measure $\beta$-galactosidase activity $P$. putida DOT-T1E cells harbouring $\mathrm{pJ} L:: \mathrm{P}_{\text {mCp } T}$ were grown overnight at $30^{\circ} \mathrm{C}$ in $\mathrm{LB}$ and $10 \mu \mathrm{g} \mathrm{ml}^{-1}$ tetracycline. Cultures were then diluted 100 -fold in the same medium supplemented or not with $1.5 \mathrm{mM}$ toluene. When the cultures reached a turbidity of $0.8 \pm 0.05$ at $600 \mathrm{~nm}, \beta$-galactosidase activity was determined in permeabilized whole cells, and activity was expressed in Miller units.

\section{Generation of the mcpT mutant in pGRT1}

The mcpT-1 mutant (ORF72:: $2 \mathrm{Km}$ ) was generated by amplification of a 615 bp fragment from $P$. putida DOT-T1E using oligonucleotides Mcp-1 and Mcp-2as described by Molina et al. (2011). DNA was extracted from the gel (Qiagen gel extraction kit, Qiagen) and ligated into $\mathrm{PMBL}_{1}-\mathrm{T}$ plasmid (Dominion MBL). The resulting plasmid was cut with Kpnl, and the insert was extracted from the gel and ligated into the pCHESI vector (Llamas et al., 2003), which had been previously digested with $\mathrm{Kpnl}$ and dephosphorylated. The plasmid was subsequently electroporated into $P$. putida DOT-T1E and transconjugants selected on LB with kanamycin. For con-

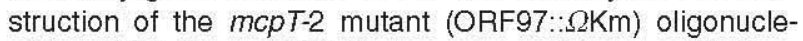
otides MCP-Eco y MCP-Bam were used for amplification. The amplicon was cut with EcoRI and BamHI and ligated into the pCHESI vector previously cut with the same enzymes; and the resulting plasmid was electroporated into P. putida DOT-T1E or the mcpT1 mutant. Resulting clones were checked for the appropriate insertion by Southern blot.

\section{Generation of plasmid $p J L-M c p T$ and complementation of the mutant}

To generate plasmid pJL-McpT, the mcp T ORF-1 (the mcpT that possesses the GAG codon for $E$ ) was amplified from $P$. putida DOT-T1E using mcPTF (5'-CACATTGAATTCTTAGAT AATGAGGTGACAC-3') and mcpTR (5'-TTCAGAGGAT CCCTAAAGACGGAACATG- $3^{\prime}$ ) primers, containing $\overline{\text { EcoRI }}$ and BamHI sites respectively (underlined). The mcpTF primer also contains a stop codon for the termination of the plasmid upstream encoded protein translation. The PCR was performed with EuroTaq polymerase (EuroTaq clone). The desired PCR product (1703 bp) was digested with EcoRI and $\mathrm{BamHI}$ and then cloned into the broad-host-range plasmid pBBRMCS-5 cleaved using the same enzymes, resulting in the pJL-mcpT plasmid. The plasmid was sequenced to confirm the presence of the desired DNA.

To express mcpT in E. coli BL21 Start ${ }^{\mathrm{TM}}$ the mcpT gene was cloned in pET20D-TOPO (Invitrogen). McpT expression was induced by adding $0.1 \mathrm{mM}$ IPTG to the bacterial culture at an $\mathrm{OD}=0.6$. Induced bacterial cultures were allowed to grow at $18^{\circ} \mathrm{C}$ overnight and cells were harvested by centrifugation at $4000 \mathrm{~g}$ for $20 \mathrm{~min}$ at $4^{\circ} \mathrm{C}$ and frozen at $-80^{\circ} \mathrm{C}$. Crude McpT-enriched membranes were prepared by thawing cell pellets on ice, resuspending them in ice cold Hepes buffer $30 \mathrm{mM} \mathrm{pH} \mathrm{7,100} \mathrm{mM} \mathrm{NaCl}$ containing EDTA-free protease inhibitor cocktail (Roche) and $100 \mathrm{U}$ benzonase (Roche), followed by French press treatment ( 1100 p.s.i., three passages per sample). The homogenized cells were first centrifuged at $4000 \mathrm{~g}$ for $15 \mathrm{~min}$ at $4^{\circ} \mathrm{C}$, the pellet discarded and the supernatant centrifuged at $50.000 \mathrm{~g}$ for $1 \mathrm{~h}$ at $4^{\circ} \mathrm{C}$. The resulting pellet of crude McpT-enriched membranes was resuspended in small aliquots of Hepes buffer $10 \%$ sucrose, flash frozen in liquid nitrogen and stored at $-80^{\circ} \mathrm{C}$. Aliquots of McpTenriched membranes were thawed just before each methylation experiments. To generate mock membranes which do not contain McpT, this procedure was repeated except that an expression plasmid which does not contain the mcpT gene was used. 
Site-directed mutagenesis to generate a single point mutant of mcpT

We generated a mutant McpT protein with a single amino acid substitution (S183P) affecting the fourth alpha-helix of the McpT-LBD using the QuikChange Mutagenesis Kit (Stratagene) following the manufacturer's protocol. The introduced mutation changed the mcpT sequence from 5'-GAGGC GTCGGCAGCT- 3 ' to 5 '-GAGGCGCCGGCAGCT-3'. The mutant allele was cloned into plasmid pJL-McpTS183P and sequenced to verify the change (see Table 1).

\section{Methylation assays}

The coding sequence of ORF PP3760 coding for CheR was cloned into the expression plasmid pET28b (Novagen). The recombinant protein was expressed with an $\mathrm{N}$-terminal His tag and purified by affinity chromatography using a $5 \mathrm{ml} \mathrm{His-}$ TrapHP column (GE Healthcare). CheR methyltransferase activity was assayed as described by Stock (1984). The assays and were conducted with McpT containing membranes and mock membranes. Briefly, equal amounts of membranes were incubated with $4 \mu \mathrm{M}$ of purified CheR, $100 \mu \mathrm{M}$ S-adenosyl-[methyl- ${ }^{3} \mathrm{H}$ ]-methionine $\left({ }^{3} \mathrm{H}-\mathrm{SAM}\right.$ : $0.83 \mu \mathrm{Ci}$ per sample), an aliquot of crude cytosolic extract of $P$. putida KT2440 (final protein concentration $5 \mathrm{mg} \mathrm{ml}^{-1}$ ), and toluene (final concentration of $1 \mathrm{mM}$ ). The final mix volume per sample was $100 \mu \mathrm{l}$ in buffer Hepes $10 \mathrm{mM}, \mathrm{NaCl}$ $100 \mathrm{mM}$. This mix was incubated at $30^{\circ} \mathrm{C}$ and aliquots were removed at regular intervals. The reaction was stopped by adding $500 \mu \mathrm{l}$ ice-cold $10 \%$ ( $\mathrm{V} / \mathrm{V}$ ) acetic acid.

In order to quantify protein methylation in these samples, it was applied the vapour-phase equilibrium procedure described by Campillo and Ashcroft (1982). Samples were washed twice with ice-cold $10 \%(\mathrm{~V} / \mathrm{N})$ acetic acid, and the pellet resuspended in $200 \mu \mathrm{l} 1 \mathrm{~N} \mathrm{NaOH}$. Each samplecontaining tube was placed opened into a $7 \mathrm{ml}$ liquid scintillation vial containing $2.4 \mathrm{ml}$ of scintillation fluid. The vial was closed and incubated overnight at $37^{\circ} \mathrm{C}$ without shaking. The average recovery of ${ }^{3} \mathrm{H}$-methanol was more than $90 \%$, and was quantified the following day using a scintillation counter. Methylation assays of mock membranes revealed a very minor activity and all measurement of McpT containing membranes were corrected with these controls.

\section{Acknowledgements}

Work was supported by FEDER grants P09-RNM-4509 $\mathrm{BIO} 2006-05699$, BIO2010-17227 and $\mathrm{BIO} 2010-16937$ from the Spanish Ministry of Science and Innovation. FEDER grants CVI-3010 and CVI1912 from the Junta de Andalucía (the Regional Government of Andalusia) and the EC BACSIN project FP7-KBBE-2007-1. We thank the BBVA Foundation for financing part of this research programme.

\section{References}

Adler, J. (1973) A method for measuring chemotaxis and use of the method to determine optimum conditions for chemotaxis by Escherichia coli. J Gen Microbiol 74: 77-91.
Alexander, R.P., and Zhulin, I.B. (2007) Evolutionary genomics reveals conserved structural determinants of signalling and adaptation in microbial chemoreceptors. ProC Natl Acad Sci USA 104: 2885-2890.

Alexandre, G., Greer-Phillips, S., and Zhulin, I.B. (2004) Ecological role of energy taxis in microorganisms. FEMS Microbiol Rev 28: 113-126.

Aono, S., Kato, T., Matsuki, M., Nakajima, H., Ohta, T., Uchida, T., and Kitagawa, T. (2002) Resonance raman and ligand binding studies of the oxygen-sensing signal transducer protein HemAT from Bacillus subtilis. $J$ Biol Chem 277: 13528-13538.

Bhushan, B., Halasz, A., Thiboutot, S., Ampleman, G., and Hawari, J. (2004) Chemotaxis-mediated biodegradation of cyclic nitramine explosives RDX, HMX, and CL-20 by Clostridium sp. EDB2. Biochem Biophys Res Commun 316: 816-821.

Boukhvalova, M.S., Dahlquist, F., and Stewart, R.C. (2002) CheW binding interactions with CheA and Tar: importance for chemotaxis signalling in Escherichia coli. $J$ Biol Chem 277: 22251-22259.

Brooun, A., Bell, J., Freitas, T., Larsen, R.W., and Alam, M. (1998) An archaeal aerotaxis transducer combines subunit I core structures of eukariotic cytochrome $c$ oxidase and eubacterial methyl-accepting chemotaxis proteins. J Bacteriol 180: 1642-1646.

Busch, A., Guazzaroni, M.E., Lacal, J., Ramos, J.L., and Krell, T. (2009) The sensor kinase TodS operates by a phosphorelay mechanism involving two autokinase domains. J Biol Chem 284: 10353-10360.

Busch, A., Lacal, J., Martos, A., Ramos, J.L., and Krell, T. (2007) Bacterial sensor kinase TodS interacts with agonistic and antagonistic signals. Proc Nat Acad Sci USA 104: 13774-13779.

Campillo, J.E., and Ashcroft, S.J.H. (1982) Protein carboxymethylation in rat islets of Langerhans. FEBS Lett 138: 71-75.

Chervitz, S.A., and Falke, J.J. (1996) Molecular mechanism of transmembrane signalling by the aspartate receptor: a model. Proc Natl Acad Sci USA 93: 2545-2550.

Cserzö, M., Wallin, E., Simon, I., von Heijne, G., and Elofsson, A. (1997) Prediction of Transmembrane alpha-helices in prokaryotic membrane proteins: the dense alignment surface method. Prot Eng 79: 681-686.

Delalez, N., and Armitage, J.P. (2009) Parts exchange: turing the flagellar motor to fit the conditions. Mol Microbiol 71: 807-810.

Deleage, G., Blanchet, C., and Geourjon, C. (1997) Protein structure prediction. Implications for the biologist. Biochimie 79: 681-686.

Gordillo, F., Chávez, F.P., and Jerez, C.A. (2007) Motility and chemotaxis of Pseudomonas sp. B4 towards polychlorobiphenyls and chlorobenzoates. FEMS Microbiol Ecol 60: 322-328.

Grimm, A.C., and Harwood, C.S. (1997) Chemotaxis of Pseudomonas spp. to the polyaromatic hydrocarbon naphthalene. Appl Environ Microbiol 63: 4111-4115.

Grimm, A.C., and Harwood, C.S. (1999) NahY, catabolic plasmid-encoded receptor required for chemotaxis of Pseudomonas putida to the aromatic hydrocarbon naphthalene. J Bacteriol 181: 3310-3316. 
Guazzaroni, M.E., Krell, T., Felipe, A., Ruiz, R., Meng, C., Zhang, X., et al. (2005) The multidrug efflux regulator TtgV recognizes a wide range of structurally different effectors in solution and complexed with target DNA: evidence from isothermal titration calorimetry. J Biol Chem 280: 2088720893.

Hazelbauer, G.L., Falke, J.J., and Parkinson, J.S. (2008) Bacterial chemoreceptors: high-performance signaling in networked arrays. Trends Biochem Sci 33: 9-19.

Iwaki, H., Muraki, T., Ishihara, S., Hasegawa, Y., Rankin, K.N., Sulea, T., et al. (2007) Characterization of a pseudomonad 2-nitrobenzoate nitroreductase and its catabolic pathway-associated 2-hydroxylaminobenzoate mutase and a chemoreceptor involved in 2-nitrobenzoate chemotaxis. J Bacteriol 189: 3502-3514.

Kanaly, R.A., and Harayama, S. (2010) Advances in the field of high-molecular-weight polycyclic aromatic hydrocarbon biodegradation by bacteria. Microb Biotechnol 3: 136164.

Kovach, M.E., Elzer, P.H., Hill, D.S., Robertson, G.T., Farris, M.A., Roop, R.M., II, and Peterson, K.M. (1995) Four new derivatives of the broad-host-range cloning vector pBBR1MCS, carrying different antibiotic-resistance cassettes. Gene 166: 175-176.

Kristich, C.J., and Ordal, G.W. (2004) Analysis of chimeric chemoreceptors in Bacillus subtillis reveals a role for CheD in the function of the McpC HAMP domain. J Bacteriol 186: 5950-5955.

Lacal, J., Busch, A., Guazzaroni, M.E., Krell, T., and Ramos, J.L. (2006) The TodS/TodT two-component regulatory system recognizes a wide range of effectors and works with DNA-bending proteins. Proc Nat Acad Sci USA 103: 8191-8196.

Lacal, J., Alfonso, C., Liu, X., Parales, R.E., Morel, B., Conejero-Lara, F., et al. (2010a) Identification of a chemoreceptor for TCA cycle intermediates: differential chemotactic response towards receptor ligands. $J$ Biol Chem 285: 23126-23136.

Lacal, J., García-Fontana, C., Muñoz-Martínez, F., Ramos, J.L., and Krell, T. (2010b) Sensing of environmental signals: classification of chemoreceptors according to size of their ligand binding regions. Environ Microbiol 12: 28732884.

Lai, W.C., Beel, B.D., and Hazelbauer, G.L. (2006) Adaptational modification and ligand occupancy have opposite effects on positioning of the transmembrane signalling helix of a chemoreceptor. Mol Microbiol 61: 1081-1090.

Liu, X., and Parales, R.E. (2009) Bacterial chemotaxis to atrazine and related s-triazines. Appl Environ Microbiol 75: 5481-5488.

Llamas, M.A., Ramos, J.L., and Rodríguez-Herva, J.J. (2003) Transcriptional organization of the Pseudomonas putida tol-oprL Genes. J Bacteriol 185: 184-195.

Marx, R.B., and Aitken, M.D. (2000) Bacterial chemotaxis enhances naphthalene degradation in a heterogeneous system. Environ Sci Technol 34: 3379-3383.

Miyakoshi, M., Shintani, M., Terabayashi, T., Kai, S., Yamane, H., and Nojiri, H. (2007) Transcriptome analysis of Pseudomonas putida KT2440 harboring the completely sequenced IncP-7 plasmid pCAR1. J Bacteriol 189: 68496860 .
Molina, L., Duque, E., Gómez, M.J., Krell, T., Lacal, J., García-Puente, A., et al., (2011) The pGRTI plasmid of Pseudomonas putida DOT-TIE encodes functions relevant for survival under harsh conditions in the environment. Environ Microbiol (in press): doi: 10.1111/j.1462-2920. 2011.02492.x.

Nielsen, M., Lundegaard, C., Lund, O., and Petersen, T.N. (2010) CPHmodels-3.0 - remote homology modeling using structure guide sequence profiles. Nucleic Acids Res 38 (Suppl.): W576-W581.

Österberg, S., Skärfstad, E., and Shingler, V. (2010) The $\sigma$-factor FliA, ppGpp and DKs $\Delta$ coordinate transcriptional control of the aer2 gene of Pseudomonas putida. Environ Microbiol 12: 1439-1451.

Ottemann, K.M., Xiao, W., Shin, Y.K., and Koshland, D.E., Jr (1999) A piston model for transmembrane signaling of the aspartate receptor. Science 285: 1751-1754.

Pandey, G., and Jain, R.K. (2002) Bacterial chemotaxis towards environmental pollutants: role in bioremediation. Appl Environ Microbiol 68: 5789-5795.

Parales, R.E., Ditty, J.L., and Harwood, C.S. (2000) Toluenedegrading bacteria are chemotactic towards the environmental pollutants benzene, toluene, and trichloroethylene. Appl Environ Microbiol 66: 4098-4104.

Ramos, J.L., Duque, E., Huertas, M.J., and Haïdour, A. (1995) Isolation and expansion of the catabolic potential of a Pseudomonas putida strain able to grow in the presence of high concentrations of aromatic hydrocarbons. J Bacteriol 177: 3911-3916.

Rodríguez-Herva, J.J., Garcia, V., Hurtado, A., Segura, A., and Ramos, J.L. (2007) The $t$ gGHI solvent efflux pump operon of Pseudomonas putida DOT-T1E Is located on a large self-transmissible plasmid. Environ Microbiol 9: 1550-1561.

Rojas, A., Segura, A., Guazzaroni, M.E., Terán, W., Hurtado, A., Gallegos, M.T., and Ramos, J.L. (2003) In vivo and in vitro evidence shows that TtgV is the local specific regulator of the TtgGHI multidrug and solvent efflux pump of Pseudomonas putida. I Bacteriol 185: 47554763.

Stock, J.B. (1984) The protein carboxy methyltransferase involved in Escherichia coli and Salmonella typhimurium chemotaxis. Methods Enzymol 106: 310-321.

Taguchi, K., Fukutomi, H., Kuroda, A., Kato, J., and Ohtake, H. (1997) Genetic identification of chemotactic transducers for amino acids in Pseudomonas aeruginosa. Microbiology 143: 3223-3229.

Timmis, K.N. (2002) Pseudomonas putida: a cosmopolitan opportunist par excellence. Environ Microbiol 4: 779-781.

Ulrich, L.E., and Zhulin, I.B. (2005) Four-helix bundle: a ubiquitous sensory module in prokaryotic signal transduction. Bioinformatics 21 (Suppl. 3): iii45-iii48.

Wadhams, G.H., and Armitage, J.P. (2004) Making sense of it all: bacterial chemotaxis. Nat Rev Mol Cell Biol 5: 10241037.

Yano, H., Garruto, C.E., Sota, M., Ohtsubo, Y., Nagata, Y., Zylstra, G.J., et al. (2007) Complete sequence determination combined with analysis of transposition/site-specific recombination events to explain genetic organization of IncP-7 TOL plasmid pWW53 and related mobile genetic elements. J Mol Biol 369: 11-26. 
Yeh, J.I., Biemann, H.P., Privé, G.G., Pandit, J., Koshland, D.E. Jr, and Kim, S.H. (1996) High-resolution structures of the ligand binding domain of the wild-type bacterial aspartate receptor. J Mol Biol 262: 186-201.

Zhulin, I.B. (2001) The superfamily of chemotaxis transduces: from physiology to genomics and back. Adv Microb Physiol 45: 157-198.

\section{Supporting information}

Additional Supporting Information may be found in the online version of this article:

Fig. S1. Physical map of the pGRT1 plasmid. The entire sequence of pGRT1 was determined by shotgun sequencing of the plasmid using the 454 technology. Sequence information can be accessed at GenBank (Molina et al., 2011).

Fig. S2. Supplementary information on McpT.

A. Protein sequence.

B. Topology and domain annotation of McpT. Analysis of the McpT sequence by DAS (Cserzö et al., 1997) revealed clearly the presence of two transmembrane regions, Tm1 and Tm2. The domain which is flanked by both $\mathrm{Tm}$ regions is therefore located in the periplasm. This domain, which remains unannotated in InterPro, is likely to correspond to the sensor domain of McpT. Analysis of the McpT sequence by InterPro shows that the cytoplasmic part of McpT is composed of a HAMP linker domain (InterPro IPR003660) and a MCP signal domain (InterPro IPR004089).
C. Secondary structure prediction of the probable sensor domain of McpT. Prediction was carried out using the consensus method (Deleage et al., 1997). Shown is the secondary structure prediction by four individual programs (DSC, GOR4, PHD and SOPMA) as well as the consensus: $h$ : $\alpha$-helix, c: coil, t: turn, e: $\beta$-strand. This prediction reveals that this domain is likely to be composed of a series of $\alpha$-helices. Ser183, which was subjected to site-directed mutagenesis, is located in the final helix of the domain and is highlighted in green.

D. Three-dimensional model of the probable sensor domain of McpT. The model was created using the $\mathrm{CPH}$ models algorithm (Nielsen et al., 2010) and the structure of the ligand binding domain of the Tsr chemoreceptor (pdb: 2D4U, unpublished) as template. The Tsr sensor domain belongs to the TarH (InterPro IPR003122) family of proteins. The model is composed of 4 helices which are coloured differently. The sequence similarity of McpT to the Tsr sensor domain and the 4-helix bundle arrangement of the model indicate that the McpT periplasmic domain is likely to be a TarH domain. It has been mentioned previously that existing bioinformatic tools to annotate TarH domains are little efficient due to the large sequence diversity within this family of proteins (Ulrich and Zhulin, 2005). 